\title{
Existence result for critical Klein-Gordon-Maxwell system involving steep potential well
}

\author{
Canlin Gan ${ }^{1}$ \\ ${ }^{1}$ Xidian University
}

December 10, 2021

\begin{abstract}
This paper deals with the following system \begin\{equation* } \} \backslash \text { left } \backslash \{ \backslash \text { begin } \text { aligned } \} \& \{ - \backslash \text { Delta } \mathrm { u } + ( \backslash \text { lambda } \mathrm { A } ( \mathrm { x } ) + 1 ) \mathrm { u } $(2 \backslash$ omega $+\backslash$ phi $) \backslash$ phi $\left.u=\backslash \operatorname{mu~f}(\mathrm{u})+\mathrm{u}^{\wedge}\{5\}\right\}, \& \&\left\{\backslash\right.$ quad $\mathrm{x} \backslash$ in $\left.\backslash \operatorname{mathbb}\{\mathrm{R}\}^{\wedge}\{3\}\right\}, \backslash \backslash \&\left\{\backslash\right.$ Delta $\backslash$ phi $=(\backslash$ omega $+\backslash$ phi $\left.) \mathrm{u}^{\wedge}\{2\}\right\}$, $\& \&\left\{\backslash\right.$ quad $\mathrm{x} \backslash$ in $\left.\backslash \operatorname{mathbb}\{\mathrm{R}\}^{\wedge}\{3\}\right\}, \backslash$ end $\{$ aligned $\} \backslash$ right. $\backslash$ end $\{$ equation* $\}$ where $\$ \backslash$ lambda, $\backslash$ mu $>0 \$$ are positive parameters. Under some suitable conditions on $\$ \mathrm{~A} \$$ and $\$ \mathrm{f} \$$, we show the boundedness of Cerami sequence for the above system by adopting Poho $\backslash v\{z\}$ aev identity and then prove the existence of ground state solution for the above system on Nehari manifold by using $\mathrm{Br} \backslash\{\mathrm{e}\}$ zis-Nirenberg technique, which improve the existing result in the literature.
\end{abstract}

\section{Hosted file}

gcl211.pdf available at https://authorea.com/users/450609/articles/548905-existence-resultfor-critical-klein-gordon-maxwell-system-involving-steep-potential-well 